\title{
Clincal interpretation of the anti-tuberculosis drug concentrations in diabetic and non-diabetic tuberculosis patients
}

\author{
Nilan T Jacob ${ }^{1}$ \\ Received: 16 February 2017 / Accepted: 27 March 2017 / Published online: 17 April 2017 \\ (C) Springer-Verlag Berlin Heidelberg 2017
}

Dear editor,

The anti-tuberculosis drug concentrations in tuberculosis patients with and without diabetes mellitus were studied by AK Hemanth Kumar et al., and the results were published recently [1]. With diabetes mellitus being an established factor increasing the risk of treatment failure, relapse, and death in tuberculosis patients [2], it is necessary to investigate the possible mechanisms behind the association.

The authors' effort to address the issue by investigating the variability in plasma drug concentrations in diabetic and nondiabetic tuberculosis patients though appreciable has a number of shortcomings. Sixteen percent of the patients in the TB + DM group were labelled diabetic based on a single random blood glucose estimation on the study day, without proper elicitation of history for symptoms of hyperglycemia; this is in violation of the diagnostic criteria for diabetes mellitus [3]. In estimating the plasma drug concentrations, a multiple timepoint sample collection aimed at demonstrating the AUC would have been more informative and robust rather than a single time point sample collection [4]. Isoniazid metabolism and subsequently the plasma drug concentrations are known to be influenced by the acetylator status of the individual, but this key association has been neglected in the study [5].

The above Letter to the Editor was sent to the authors of the paper that this Letter comments on, but unfortunately, we have not had a response commentary from them. / Editor

Nilan T Jacob

nilantjacob20@gmail.com

1 Jawaharlal Institute of Postgraduate Medical Education and Research (JIPMER), Puducherry, India
In the sample size estimation, selection of a higher true difference in the plasma drug concentrations could have led to a reduction in the sample size without affecting the results. The coefficient value for association between gender and pyrazinamide concentrations $(-2.363)$ has not been specified for the gender type. The correlation coefficient at $r=-0.09$ for isoniazid and $r=-0.092$ for pyrazinamide demonstrate a very weak correlation despite the statistical significance. Moreover, the plasma drug concentrations for both isoniazid and pyrazinamide show only a relative variability, and the drug concentrations are within the normal therapeutic range for the respective drugs. This makes for a very weak or rather non-existent clinical implication for the results.

In spite of failing to add new information on the mechanisms behind the association of diabetes mellitus and poorer outcome in tuberculosis patients, the study brings to light the possibility of exploring the pharmacokinetics of isoniazid and pyrazinamide in the setting of elevated blood glucose levels.

\section{References}

1. Hemanth Kumar AK, Chandrasekaran V, Kannan T et al (2017) Anti-tuberculosis drug concentrations in tuberculosis patients with and without diabetes mellitus. Euro J Clin Pharmacol 73:65-70

2. Baker MA, Harries AD, Jeon CY et al (2011) The impact of diabetes on tuberculosis treatment outcomes: a systematic review. BMC Med 9:81

3. American Diabetes Association (2015) Diabetes Care 38(Supplement 1):S8-S16

4. Burhan E, Ruesen C, Ruslami R et al (2013) Antimicrob Agents Chemother 57:3614-3619

5. Weber WW, Hein DW (1979) Clinical pharmacokinetics of isoniazid. Clin Pharmacokinet 4:401-422 